\title{
Effects of long-term treatment with pioglitazone on cognition and glucose metabolism of PS1-KI, $3 \times T g-A D$, and wild-type mice
}

\author{
F Masciopinto ${ }^{1,2,9}$, N Di Pietro ${ }^{3,9}$, C Corona ${ }^{1,2}$, M Bomba ${ }^{1,2}$, C Pipino ${ }^{3}$, M Curcio ${ }^{4}$, A Di Castelnuovo ${ }^{5}$, D Ciavardelli ${ }^{1,6}$, E Silvestri ${ }^{4}$, \\ LMT Canzoniero ${ }^{4}$, I Sekler ${ }^{7}$, A Pandolfi $^{3}$ and SL Sensi ${ }^{\star, 1,2,8}$
}

In this study, we investigated the effects of long-term (9-month) treatment with pioglitazone (PIO; $20 \mathrm{mg} / \mathrm{kg} / \mathrm{d})$ in two animal models of Alzheimer's disease (AD)-related neural dysfunction and pathology: the $\mathrm{PS} 1-\mathrm{KI}_{\mathrm{M} 146 \mathrm{~V}}$ (human presenilin-1 ${ }^{\mathrm{M} 146 \mathrm{~V}}$ knockin mouse) and 3xTg-AD (triple transgenic mouse carrying $A D$-linked mutations) mice. We also investigated the effects on wildtype (WT) mice. Mice were monitored for body mass changes, fasting glycemia, glucose tolerance, and studied for changes in brain mitochondrial enzyme activity (complexes I and IV) as well as energy metabolism (lactate dehydrogenase (LDH)). Cognitive effects were investigated with the Morris water maze (MWM) test and the object recognition task (ORT). Behavioral analysis revealed that PIO treatment promoted positive cognitive effects in PS1-KI female mice. These effects were associated with normalization of peripheral gluco-regulatory abnormalities that were found in untreated PS1-KI females. PIO-treated PS1-KI females also showed no statistically significant alterations in brain mitochondrial enzyme activity but significantly increased reverse LDH activity.PIO treatment produced no effects on cognition, glucose metabolism, or mitochondrial functioning in 3xTgAD mice. Finally, PIO treatment promoted enhanced short-term memory performance in WT male mice, a group that did not show deregulation of glucose metabolism but that showed decreased activity of complex I in hippocampal and cortical mitochondria. Overall, these results indicate metabolically driven cognitive-enhancing effects of PIO that are differentially gender-related among specific genotypes.

Cell Death and Disease (2012) 3, e448; doi:10.1038/cddis.2012.189; published online 20 December 2012

Subject Category: Neuroscience

Glucose dysmetabolism is a critical promoter of brain impairment. $^{1}$

Type 2 diabetes mellitus (T2DM), a disorder of peripheral glucose regulation, is also associated with cognitive decline. ${ }^{2}$ Aging enhances the negative impact of T2DM on cognitive functions. ${ }^{3}$ Interestingly, emerging evidence in the two past decades has shown that T2DM and Alzheimer's Disease (AD) share common pathogenic mechanisms, ${ }^{4}$ such as impaired glucose metabolism, increased oxidative stress, insulin resistance, and amyloidogenesis. Increased expression in the central nervous system of insulin receptors, not coupled with changes in Tyr-kinase activity, can lead to impairment of insulin signaling and is found in AD and T2DM patients. ${ }^{5}$

In the brain, insulin signaling is not exclusively involved in metabolic processes but also has a role in modulating neurotrophic and neuroendocrine functions. ${ }^{6}$ The hippocampus, a strategic area for cognition, contains substantial amounts of immunoreactive insulin and insulin receptors, ${ }^{7}$ while hippocampal neurons have been shown to release insulin under depolarizing conditions. ${ }^{8}$ Strengthening the importance of the insulin-cognition link, insulin receptordefective mice have been reported to develop cognitive impairment. ${ }^{9}$ Moreover, intranasal insulin administration has been shown to improve cognitive functions in diabetic and non-diabetic mice ${ }^{10,11}$ as well as in humans, ${ }^{12}$ an effect that is achieved without producing significant alterations in blood glucose levels. ${ }^{13}$

Treatments aimed at improving insulin signaling have also been investigated for their neuroprotective potential in AD and in ischemic brain damage. ${ }^{14}$ Thiazolidinediones (TZDs) act as

\footnotetext{
${ }^{1}$ Molecular Neurology Unit-Center of Excellence on Aging (Ce.S.I.), University 'G. d'Annunzio', Chieti-Pescara, Italy; ${ }^{2}$ Department of Neuroscience and Imaging, University 'G. d'Annunzio', Chieti-Pescara, Italy; ${ }^{3}$ Department of Experimental and Clinical Sciences, University 'G. d'Annunzio' and Ce.S.I., Chieti-Pescara, Italy; ${ }^{4}$ Department of Biological and Environmental Science, University of Sannio, Benevento, Italy; ${ }^{5}$ Environmental and genetic epidemiology laboratory, Research Laboratories, FRC 'Giovanni Paolo II', Campobasso, Italy; ${ }^{6}$ School of Engineering, Architecture, and Motor Science, 'Kore' University, Enna, Italy; ${ }^{7}$ Department of Physiology, School of Health Sciences, Ben-Gurion University of the Negev, Beer-Sheva, Israel and ${ }^{8}$ Departments of Neurology and Pharmacology, University of California-Irvine, Irvine, CA, USA

${ }^{*}$ Corresponding author: SL Sensi, Molecular Neurology Unit, Ce.S.I., Via Colle dell' Ara, Chieti 66013, Italy. Tel: + $390871541544 ;$ Fax: + 390871541542 ;

E-mail: ssensi@uci.edu

${ }^{9}$ These authors contributed equally to this work.

Keywords: pioglitazone; neurodegeneration; insulin signaling; glucose metabolism; mitochondrial complex activity; LDH

Abbreviations: PIO, pioglitazone; AD, Alzheimer's disease; WT, wild type; PS1-KI, human presenilin-1 ${ }^{\mathrm{M} 146 \mathrm{~V}}$ knock-in mouse; 3xTg-AD, triple transgenic mouse carrying AD-linked mutations; MWM, Morris water maze; ORT, object recognition test for object-place task; IPGTT, intraperitoneal glucose tolerance test; PPAR $\gamma$, peroxisome proliferator-activated receptor- $\gamma$; APP, amyloid precursor protein; DI, Discrimination Index; TZD, thiazolidinedione; BN-PAGE, blue-native polyacrylamide gel electrophoresis; LDH, lactate dehydrogenase.

Received 12.9.12; revised 8.11.12; accepted 15.11.12; Edited by A Verkhratsky
} 
glucose-lowering drugs mainly through the activation of the nuclear receptor peroxisome proliferator-activated receptor- $\gamma$ (PPAR $\gamma$ ) and have been tested as neuroprotective agents.

PPAR $\gamma$ receptors are broadly distributed in the central nervous system ${ }^{15}$ and, in the past decade, accumulating evidence has shown that therapeutic targeting of these receptors can prevent neuronal death by inducing a reduction of oxidative stress and inflammation. ${ }^{16}$ Recent evidence indicates that improvement of insulin signaling through PPAR $\gamma$ agonists contributes to synaptic protection against damages induced by soluble $\beta$-amyloid $(\mathrm{A} \beta)$-derived diffusible ligands. ${ }^{17,18}$

Interestingly, brain extracts from $A D$ patients show decreased expression of PPAR $\gamma$ and decreased binding to the PPAR $\gamma$ responsive element in the gene promoter region of the $\beta$-site APP (amyloid precursor protein)-cleaving enzyme (BACE1), the rate-limiting enzyme involved in the processing of the APP. ${ }^{19}$ Overexpression of PPAR $\gamma \mathrm{s}$, as well as treatment with non-steroidal anti-inflammatory drugs and PPAR $\gamma$ activators reduces BACE1 gene promoter activity and promotes decreased levels of $A \beta .^{19,20}$

$A D$ development is associated with mitochondrial impairment, a phenomenon that is thought to precede $\mathrm{A} \beta$ and tau protein aggregation. ${ }^{21}$ PPAR $\gamma$ ligands interact with mitochondrial proteins, ${ }^{22}$ decrease mitochondrial reactive oxygen species (mtROS) production, and also promote mitochondrial biogenesis and respiration. ${ }^{23}$

In this study, we investigated TZD-related cognitive and metabolic effects in two models of AD-related neuronal dysfunction and pathology: the $\mathrm{PS} 1-\mathrm{KI}_{\mathrm{M} 146 \mathrm{~V}}$ (human presenilin-1 $1_{M 146 V}$ knock-in mouse) mouse that shows brain dysfunctions developed in $\mathrm{A} \beta$-independent way and the $3 \times T g-A D$ (triple transgenic mouse carrying AD-linked mutations) mice that show amyloid and tau-dependent pathology and cognitive decline.

$\mathrm{PS} 1-\mathrm{KI}_{\mathrm{M} 146 \mathrm{~V}}$ mice carry a mutation in presenilin-1 (PS1), the catalytic subunit of the $\gamma$-secretase complex (another key enzyme involved in APP cleavage and A $\beta$ production). PS1 mutations have been linked to development of familial $A D$ (FAD). Despite the lack of plaque deposits, PS1 mutant mice exhibit a variety of pathological and functional changes that include: impaired hippocampal neurogenesis due to altered Notch signaling, delayed axonal transport, appearance of abnormally phosphorylated neurofilament-associated proteins, increased brain levels of lipid and protein peroxidation products as well as enhanced expression of components of the oxidative phosphorylation (for review, see Elder et al. ${ }^{24}$ ). Young PS1-mutant mice show enhanced long-term potentiation, but this transitory positive condition disappears with aging. ${ }^{25}$ Thus, this model seems suitable to investigate alterations that underlie the development of age-dependent cognitive deficits in an amyloid-independent fashion.

3xTg-AD mice overexpress mutant PS1, APP, and hyperphosphorylated $\operatorname{tau}^{26}$ and are considered an excellent $A D$ model as they show $\mathrm{A} \beta$ - and tau-dependent mechanisms leading to $A D$-like neurodegeneration and cognitive decline. As control for both the groups, we used wild-type (WT) mice.

To investigate TZD-related effects, we used pioglitazone (PIO), a TZD that is particularly effective in passing the bloodbrain barrier and has been tested as neuroprotective agent. ${ }^{27}$

\section{Results}

PIO effects on short- and long-term memory. In this set of experiments, we evaluated, with the Morris water maze (MWM), short- and long-term spatial memory, two processes highly dependent on the function and plasticity of the dorsal hippocampus. ${ }^{28}$ Classical MWM scores include: time taken by the mouse to reach the location of a platform that the animal is instructed to find during the 4 days training sessions (latency) as well as the number of crosses made over the hidden platform (crosses).

In our experiments, we found that PIO-treated animals showed a statistically significant increase in body weight (a well-known side effect of the drug) that made the navigation in the maze slower, independently from changes in cognitive abilities. Thus, to evaluate cognitive effects, we considered the number of crosses. Analysis of both short- and long-term MWM data showed that all the genotypes fed with CTRL-diet performed equally well, thereby indicating that they were not having memory deficits at levels to be detected by the test.

PIO treatment did not produce effects on short-term memory in any group (Figure 1a), with the exception of male WT that showed a statistically significant increase in crosses (Figure 1a; PIO-treated male WT versus untreated WT males, $P=0.022$ ).

Analysis of long-term memory performance indicated that $\mathrm{PIO}$ treatment promoted a robust enhancement in long-term memory in PS1-KI female mice (Figure 1b; PIO-treated female PSI-KI versus untreated female PS1-KI, $P=0.030$ ); the effect of mutant genotype in the PIO-driven cognitive enhancement was also tested and a statistically significant difference was found when comparing PIO-treated female $\mathrm{PS} 1-\mathrm{KI}$ versus PIO-treated female WT (Figure $1 \mathrm{~b}, P=0.015$ ). $\mathrm{PIO}-$ dependent cognitive-enhancing effects are also genderspecific as PS1-KI male mice showed no amelioration. Finally, $\mathrm{PIO}$ treatment did not affect long-term memory performance in WT and 3xTg-AD mice.

PIO effects on object recognition task (ORT). To further evaluate cognition we used ORT, another behavioral test used to investigate the animal's ability to build spatial relationships between distal extra-maze cues that rely on correct functioning of the dorsal hippocampus. ${ }^{29}$ Some studies indicate that ORT can be more sensitive than MWM as ORT is based on a test modality that eliminates possible bias deriving from behavioral modifications that could influence the memory performance and that are connected with drug-related changes of pain perception, stress susceptibility, thermoregulation, and anxiety. ${ }^{29-31}$ Upon ORT, mice are exposed to a one-trial object-place recognition task. Animals are presented two identical objects during the familiarization phase and, the next day, one object is shifted to a novel location (probe trial). A classical parameter used to analyze ORT is the Discrimination Index (DI), an index that expresses, in percentage, the difference between exploration time spent on the object positioned in the novel location and exploration time spent on the familiar one, all divided to the total exploration time. ${ }^{32}$

Supporting the higher sensitivity of ORT in detecting subtle cognitive deficits, DI analysis showed that CTRL-diet PS1-KI 

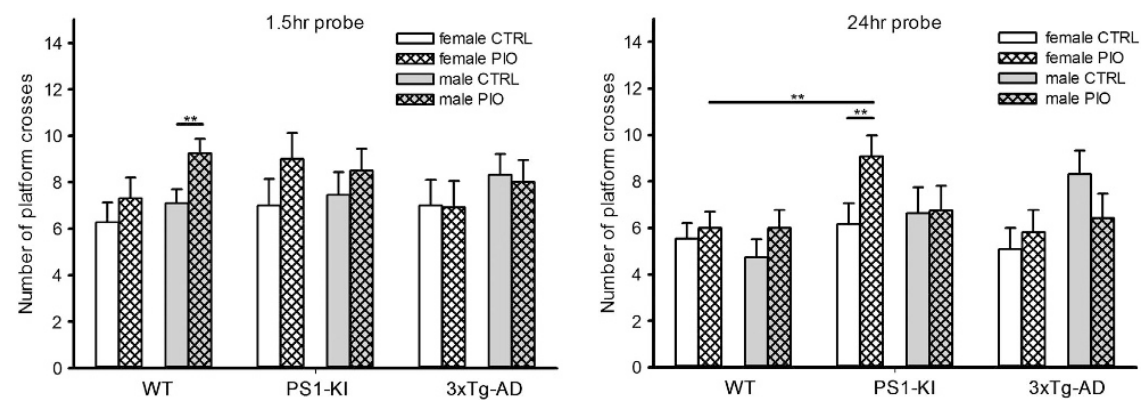

Figure 1 PIO effects on short- and long-term memory analyzed with the MWM test. Bar graphs depict MWM results evaluated in term of number of platform crosses. (a) Short-term memory. WT male mice treated with PIO and tested for effects on short-term memory ( $1.5 \mathrm{~h}$ after the last training session) showed statistically significant increases in crosses compared with CTRL-diet WT mice (PIO-diet male WT versus CTRL-diet male WT, $P=0.022)$. All other groups were not affected by PIO treatment. (b) Long-term memory. Analysis of swimming performance $24 \mathrm{~h}$ after the last training session to evaluate long-term memory showed improvement in PIO-treated PS1-KI females compared with CTRL-diet female mice (PIO-diet female PS1-KI versus CTRL-diet female PS1-KI, $P=0.030$; Right panel). The improvement is also highlighted by the comparison with PIO-treated WT females (PIO-diet female PS1-KI versus PIO-diet female WT, $P=0.015$ ). Females and males are depicted with white and gray bars, respectively; CTRL-diet is shown with open bar, PIO-diet is represented with crossed bars. Data are shown as means \pm S.E.M.; $n=12$ for each group. Statistical analysis was performed with analysis of variance for repeated measures; ${ }^{* *} 0.05 \leq P<0.01$

females were cognitively impaired when compared with WT females (Figure 2, $P=0.005$ ). PIO-treated PS1-KI females exhibited cognitive improvement as indicated by significantly increased DI scores compared with CTRL-diet PS1-KI mice (Figure 2, $P=0.049$ ). DI scores also showed a trend toward worsening of memory functions in PIO-treated male WT mice (Figure 2, $P=0.072$ ). PIO treatment did not affect memory performance of 3xTg-AD mice of both sexes.

PIO effects on body weight. PPAR $\gamma$ s regulate glucose and lipidic metabolism by modulating energy homeostasis in the adipose tissue, skeletal muscle, and liver. ${ }^{33}$ PIO has wellknown effects on body weight, and we therefore investigated weight changes occurring throughout the 9-month treatment period in PS1-KI, 3xTg-AD, and WT mice (Figure 3).

A physiological time-dependent gain in body weight occurred in all the untreated genotypes. Moreover, PS1-KI mice, both males and females, and male 3xTg-AD mice gained significantly more weight compared with WT mice (Figures $3 b$ and a upper panels, CTRL-diet female PS1-KI versus CTRL-diet female WT, $P=0.002$, and Figures $3 \mathrm{~b}$ and a lower panels, CTRL-diet male PS1-KI versus CTRL-diet male WT, $P=0.010$; Figures $3 c$ and a lower panels, CTRLdiet male 3xTg-AD versus CTRL-diet male $\mathrm{WT}, P=0.001$ ).

$\mathrm{PIO}$ treatment produced increased gain in weight in all the study groups. This effect was significantly enhanced in PS1$\mathrm{KI}$ mice when compared with PIO-treated WT mice (Figures $\mathrm{Bb}$ and a upper panels, PIO-treated female PS1-KI versus PIO-treated female WT, $P=0.004$, and Figures $3 \mathrm{~b}$ and a lower panels, PIO-treated male PS1-KI versus PIO-treated male WT, $P=0.001$ ) or to PIO-treated 3xTg-AD mice (Figures $3 c$ and $b$ upper panels, PIO-treated female PS1-KI versus PIO-treated female 3xTg-AD, $P=0.008$, and Figures $3 \mathrm{c}$ and $\mathrm{b}$ lower panels, $\mathrm{PIO}$-treated male PS1-KI versus $\mathrm{PIO}$-treated male 3xTg-AD, $P=0.010)$. Finally, $P S 1-K I$ male mice under PIO-diet gained more weight than female mice of the same genotype (Figure 3b upper and lower panels, PIO-treated male PS1-KI versus PIO-treated female PS1-KI, $P=0.028$ ).

PIO effects on glucose metabolism. To investigate glucose metabolism, we monitored changes in fasting blood

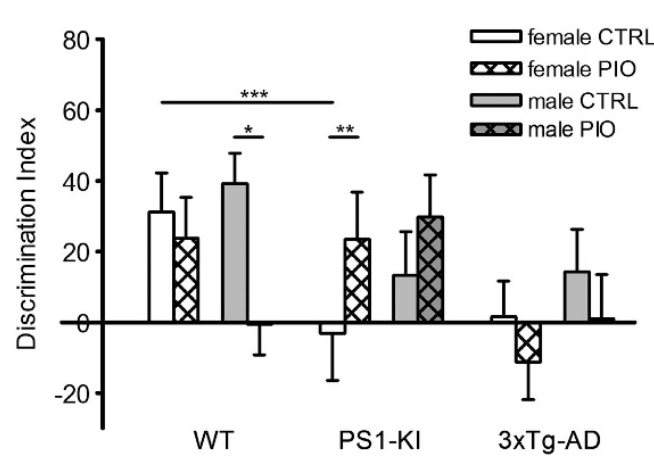

Figure 2 PIO effects on memory performance analyzed with the ORT. Bar graphs depict ORT results as DI scores evaluated $24 \mathrm{~h}$ after the familiarization trial. Compared with WT, PS1-KI females developed memory deficits (CTRL-diet female PS1-KI versus CTRL-diet female WT, $P=0.005$ ). PIO-treated PS1-KI females exhibited improvements in recognition of displaced objects as indicated by increased DI scores (PIO-diet female PS1-KI versus CTRL-diet female PS1-KI, $P=0.049)$. WT male mice treated with PIO showed a trend toward worsening of long-term memory (PIO-diet male WT versus CTRL-diet male WT, $P=0.072$ ). Females and males are depicted with white and gray bars, respectively; CTRL-diet is shown with opened bars, PIO-diet is represented with crossed bars. Data are shown as means \pm S.E.M.; $n=12$ for each group. Statistical analysis was performed with analysis of variance for repeated measures; ${ }^{*} 0.05<P \leq 0.095$; ${ }^{* *} 0.05 \leq P<0.01 ;{ }^{* \star *} P \leq 0.01$

glucose levels, glucose tolerance, and serum insulin levels during the 9-month treatment in all the groups.

Compared with WT mice, female and male PS1-KI mice undergoing CTRL diet developed signs of peripheral glucoregulatory abnormalities as indicated by increased fasting blood glucose values (CTRL-diet female PS1-KI versus CTRL-diet female WT, $P=0.020$; CTRL-diet male PS1-KI versus CTRL-diet male WT, $P=0.010)$. PIO treatment normalized this near pre-diabetic condition in female PS1-KI mice (Figure 4b upper panel, PIO-diet female PS1-KI versus CTRL-diet female PS1-KI, $P<0,001)$. PIO treatment had no effect on PS1-KI males (Figure 4b lower panel, PIO-diet male $\mathrm{PS} 1-\mathrm{KI}$ versus CTRL-diet male PS1-KI, $P=0.44$ ).

No significant changes in fasting glycemia levels were found in PIO-treated WT and 3xTg-AD mice. 
a

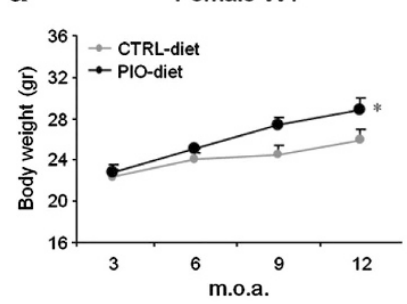

Male WT

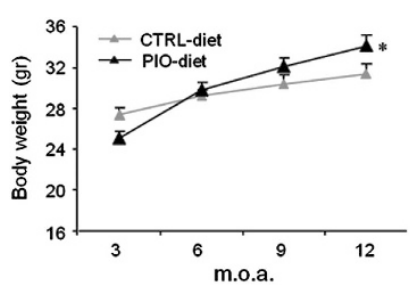

b

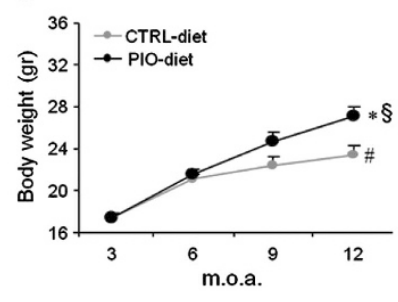

Male PS1-KI

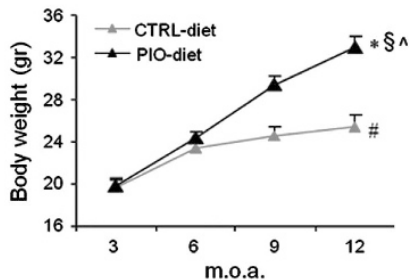

C Female $3 \times T g-A D$

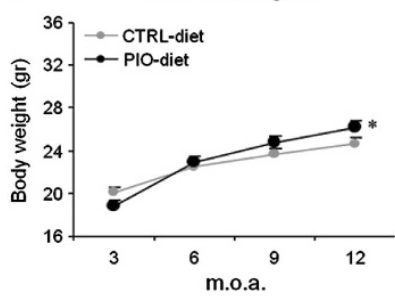

Male 3xTg-AD

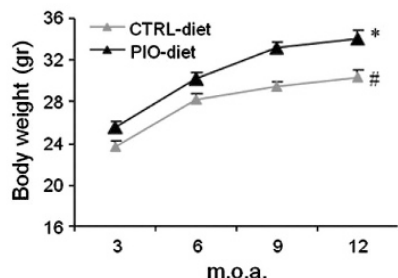

Figure 3 PIO effects on body weight. Graphs show time course of body weight changes monitored during the 9-month treatment in treated and untreated (a) WT, (b) PS1$\mathrm{KI}$, and (c) 3xTg-AD mice. Upper panels show weight changes in female mice; lower panels depict changes in males. All untreated genotypes showed a physiological timedependent gain in body weight. PS1-KI mice and male 3xTg-AD mice gained significantly more weight compared with WT mice (CTRL-diet female PS1-KI versus CTRL-diet female WT, $P=0.002$; CTRL-diet male PS1-KI versus CTRL-diet male WT, $P=0.01$; CTRL-diet male 3xTg-AD versus CTRL-diet male WT, $P=0.001$ ). PIO treatment induced a significant time-dependent increase in body weight in all the treated genotypes. Enhanced PS1-KI gain in weight was observed when comparing these animals with PIO-diet WT mice (PIO-diet female PS1-KI versus PIO-diet female WT, $P=0.004$; PIO-diet male PS1-KI versus PIO-diet male WT, $P=0.001$ ) or when comparing with PIOdiet 3xTg-AD mice (PIO-diet female PS1-KI versus PIO-diet female 3xTg-AD, $P=0.008$, and PIO-diet male PS1-KI versus PIO-diet male 3xTg-AD, $P=0.010$ ). PIO effects on PS1-KI body weight were also gender-related (PIO-diet male PS1-KI versus PIO-diet female PS1-KI, $P=0.028$ ). Data are shown as means \pm S.E.M.; $n=12$ for each group. Statistical analysis was performed with analysis of variance for repeated measures. ${ }^{*} \mathrm{PIO}$ treatment effect; ${ }^{\#}$ genotype effect; ${ }^{\S}$ gender effect. $P \leq 0.01$ in all cases

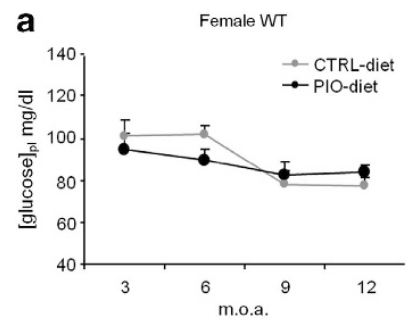

Male WT

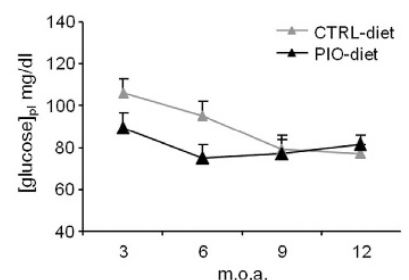

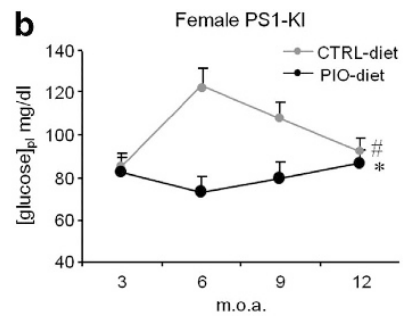

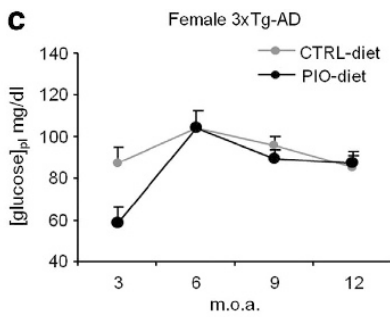

Male PS1-KI

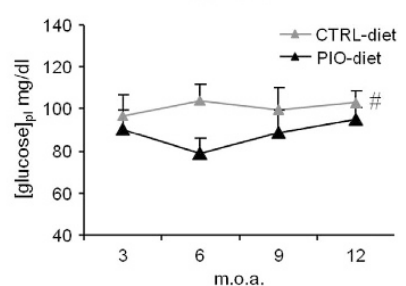

Male 3xTg-AD

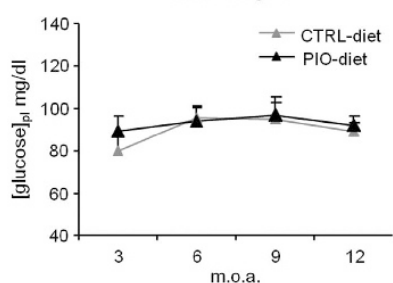

Figure 4 PIO effects on fasting glycemia. Graphs show time course of fasting blood glucose levels measured during the 9-month period in treated and untreated (a) WT, (b) PS1-KI, and (c) 3xTg-AD mice. Upper panels show results in female mice, lower panels depict findings in males. Female and male PS1-KI mice under CTRL-diet showed a significant time-dependent increase in fasting glycaemiaglycaemia when compared with WT mice (CTRL-diet female PS1-KI versus CTRL-diet female WT, $P=0.02$, and CTRL-diet male PS1-KI versus CTRL-diet male WT, $P=0.01$ ). PIO treatment induced a statistically significant reduction of fasting glycemia in female PS1-KI mice (PIO-diet female PS1-KI versus CTRL-diet female PS1-KI, $P<0.001$ ). On the contrary, PIO treatment had no effect on PS1-KI males (PIO-diet male PS1-KI versus CTRL-diet male $\mathrm{PS} 1-\mathrm{KI}, P=0.440$ ). Data are shown as means \pm S.E.M.; $n=12$ for each group. Statistical analysis was performed with analysis of variance for repeated measures. ${ }^{*} \mathrm{PIO}$

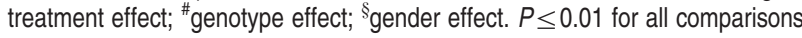

Results of the intraperitoneal glucose tolerance test (IPGTT, a test that measures glucose tolerance in mice) showed that untreated PS1-KI females exhibited a trend toward impaired glucose tolerance (CTRL-diet female PS1-KI versus CTRL-diet female WT, $P=0.064)$, a phenomenon that was not affected by PIO-treatment ( $P=0.98$; data not shown).

No significant changes in IPGTT levels were found in PIOtreated WT and 3xTg-AD mice. Serum insulin levels were not changed by drug treatment, genotypes, and gender (data not shown).

PIO effects on brain mitochondrial function. Mitochondrial dysfunctions have a pivotal role in aging, neurodegeneration, and $\mathrm{AD} .^{21,34}$

To evaluate effects of PIO treatment in our models, we investigated expression and activity of electron transport 
chain complexes I and IV in the cortex and hippocampus of the three genotypes. To that aim, we used a combination of bluenative polyacrylamide gel electrophoresis (BN-PAGE) ${ }^{35}$ and subsequent histochemical in-gel staining. This method is extensively used for the diagnosis of OXPHOS alterations and provides indirect information on mitochondrial enzymatic activity in view of the native conditions that are maintained during the analysis (i.e., the isolation of samples material and solubilization with mild detergents). Thus, it is well established that BN-PAGE combined with subsequent catalytic staining allow the preservation of enzymatic activities and the detection of respiratory complex activities/deficiencies. ${ }^{36,37}$ We do acknowledge, though, that the measurement and assessment of specific activities in reactive bands need to be considered semi-quantitative and strongly affected by the 'in gel' milieu.

As shown in Figures 5 and 6 different profiles of $\mathrm{PIO}$-driven changes were found.

Analysis in PS1-KI female mice revealed that PIO induced a trend toward reduction in the activity of complex I in the hippocampus (Figure 5, Supplementary Table S1, PIO-diet female PS1-KI versus CTRL-diet female PS1-KI, $P=0.090$ ). No expression changes were found. Expression levels of complex I were found increased in the cortex (Figure 6, Supplementary Table S2, PIO-diet female PS1-KI versus CTRL-diet female PS1-KI, $P=0.020$ ), however, no changes in activity were detected. Male PS1-KI mice responded to PIO treatment only with an increase in cortical expression of complex I (Figure 6, Supplementary Table S2, PIO-diet male PS1-KI versus CTRL-diet male PS1-KI, $P=0.018$ ), whereas no changes in activity of complexes I and IV were observed. In the hippocampus, no changes in complex activity and expression were observed.

In 3xTg-AD mice of both genders, we did not detect any change in complexes I and IV expression and activity.

In WT PIO-treated female mice, we found an agedependent decay in the expression of complexes I and IV in the hippocampus (Figure 5, Supplementary Table S1, PIOdiet female WT versus CTRL-diet female WT, $P=0.010$ and $P=0.015$, respectively) whereas, in the cortex, we only observed a decreased expression of complex I (Figure 6, Supplementary Table S2, PIO-diet female WT versus CTRLdiet female WT, $P=0.029$ ). These changes were not paralleled by alterations in complex activity in both the brain regions. In WT male mice, we found that PIO did not change expression of complexes I and IV in both tissues but

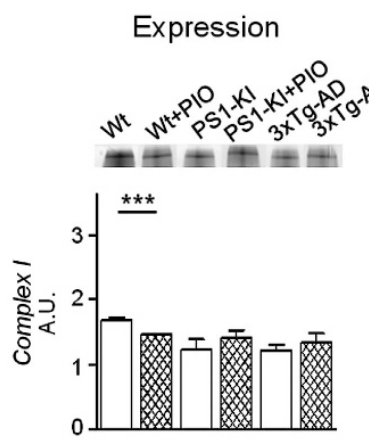

FEMALE a<smiles>COC(=O)O</smiles>

\section{HIPPOCAMPUS}

b

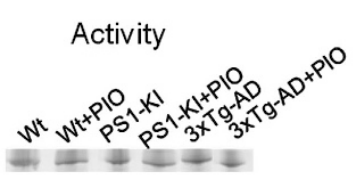

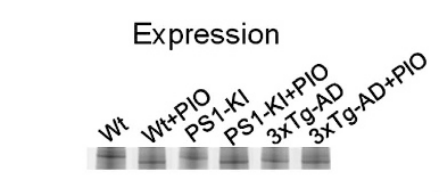

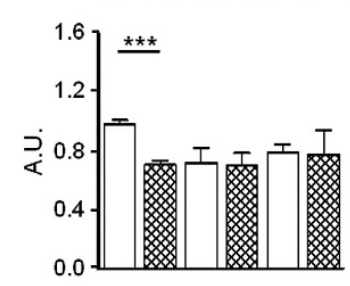

$$
\text { Expression }
$$
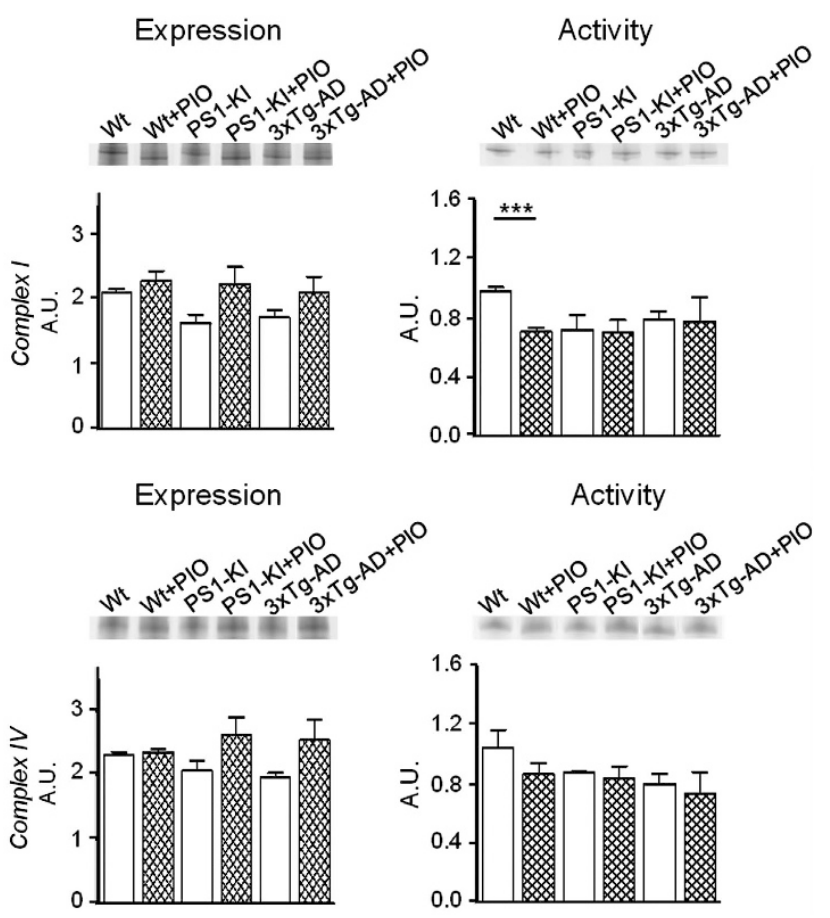

Activity

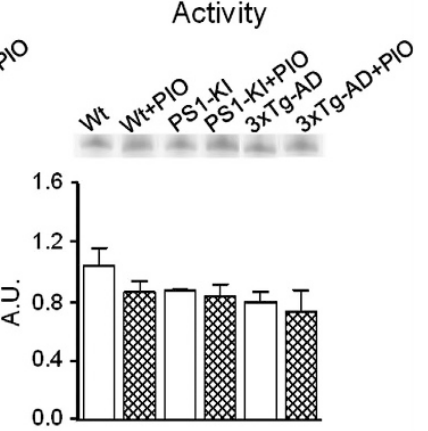

Figure $5 \mathrm{PIO}$ effects on complexes I and IV expression and activity in hippocampal mitochondria. Mitochondrial fractions from hippocampi (10 mg; wet weight) of treated and untreated animals were analyzed with BN-PAGE and subsequent histochemical in-gel staining to evaluate expression and activity of electron transport chain complexes I and IV. Panel a shows results in female mice, panel $\mathbf{b}$ shows results in males. Complex I results are in the upper part and complex IV in the lower part. For each complex, expression and activity data are shown along with representative blots. (a) PIO treatment induced significant reductions in expression of both complexes in WT animals. PIO induced a trend toward reduction in activity levels of complex I in PS1-KI mice (see also Supplementary Table S1). (b) PIO treatment induced significant decreased activity of complex I in WT animals. CTRL- and PIO-diet samples are shown with open and crossed bars, respectively. Band areas are expressed as absolute values (arbitrary units (A.U.)). Data are shown as means \pm S.E.M.; $n=4$ for each group. Statistical analysis was performed with two-tailed Student's $t$-test for unpaired comparisons between the treated and untreated groups (95\% confidence level). ${ }^{*} 0.05<P \leq 0.095 ;{ }^{* *} 0.05 \leq P<0.01 ;{ }^{* \star *} P \leq 0.01$ 


\section{Expression}

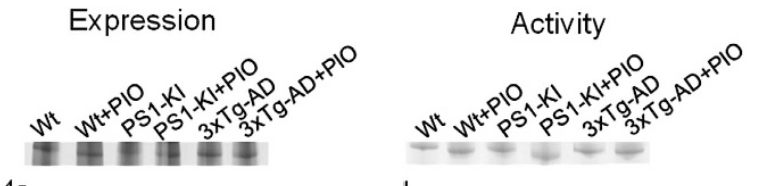
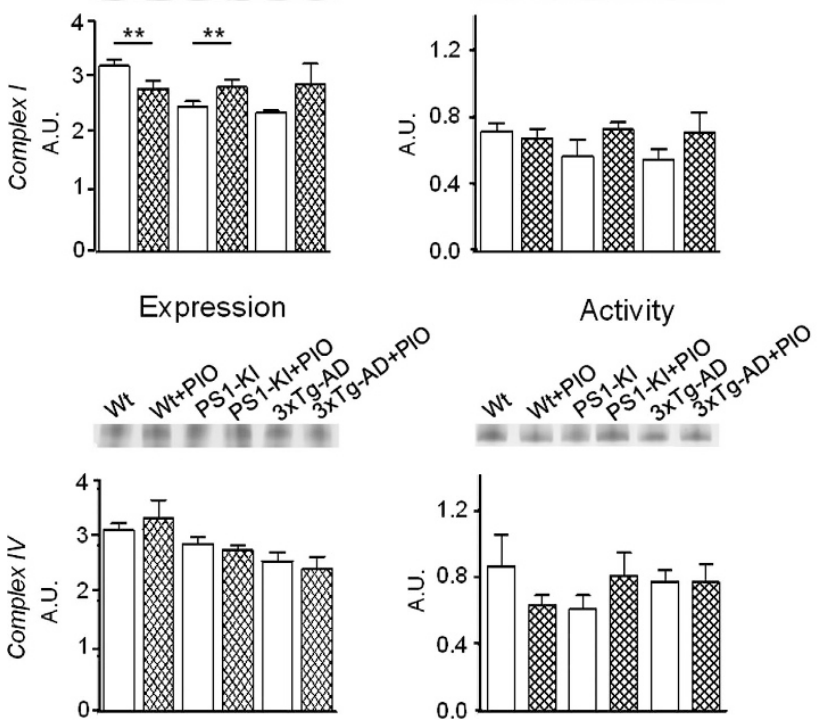

Figure 6 PIO effects on complex I and complex IV expression and activity in cortical mitochondria. Mitochondrial fractions from cortex (10 mg; wet weight) of treated and untreated animals were analyzed as in Figure 5. Panel a shows results in female mice. Panel b shows results in male mice. PIO treatment induced significantly decreased expression levels of complex I in WT female mice. Expression levels of complex I were increased in PIO-treated PS1-KI mice, both genders. Complex I activity was found significantly decreased in PIO-treated WT males. No changes were found for complex IV. CTRL- and PIO-diet samples are shown with open and crossed bars, respectively. Band areas were expressed as absolute values (A.U., arbitrary units). Data are shown as means \pm S.E.M.; $n=4$ for each group. Statistical analysis was performed with twotailed Student's $t$-test for unpaired comparisons between the treated and untreated groups $\left(95 \%\right.$ confidence level). ${ }^{*} 0.05<P \leq 0.095 ;{ }^{*} 0.05 \leq P<0.01 ;{ }^{* * \star} P \leq 0.01$

significantly decreased complex I activity in the hippocampus (Figure 5, Supplementary Table S1, PIO-diet male WT versus CTRL-diet male WT, $P=0.002$ ) and cortex (Figure 6, Supplementary Table S2, PIO-diet male WT versus CTRLdiet male WT, $P<0.01$ ).

PIO effects on brain lactate dehydrogenase (LDH) activities. To further evaluate PIO-driven changes in energy metabolism, we assayed forward ( $\left.\mathrm{LDH}_{\mathrm{Pyr} \rightarrow \mathrm{Lac}}\right)$ and reverse $\left(\mathrm{LDH}_{\mathrm{Lac} \rightarrow \text { Pyr }}\right) \mathrm{LDH}$ activities in the cytosolic fraction of brain samples obtained from treated and untreated mice of the three genotypes. This spectrophotometric assay allows estimation of brain LDH activity through measurement of relative increases or decreases of enzyme activity-linked absorbance in cytosolic samples. ${ }^{38}$

Analysis of the LDH data by three-way ANOVA indicates that PIO treatment significantly decreased forward activity in the case of female WT mice (Figure $7 a, P=0.020$ ) while a trend toward decreased forward activity was found in female PS1-KI mice (Figure 7a, $P=0.073$ ).

When analyzing reverse LDH activity, we found that PIO treatment produced significant increases in female and male WT mice (Figure 7b, $P=0.002$ and $P=0.024$, respectively) as well as in female PS1-KI mice (Figure $7 \mathrm{~b}, P=0.033$ ).

\section{Discussion}

In this study, we tested cognitive and metabolic effects triggered by long-term treatment with PIO on PS1-KI and 3xTg-AD mice along with WT mice used as controls. To our knowledge, this is the first study investigating the effects of 9month treatment with PIO on two animal models that are prone to neural and brain dysfunction.

Our MWM and ORT findings indicate that PIO treatment promoted enhanced long-term memory performance in PS1$\mathrm{KI}$ females. Interestingly, these mice developed a transitory near pre-diabetic status, ${ }^{39}$ with increased fasting glycemia at 6 months of age, a phenomenon that was counteracted by PIO. Normalization of fasting glycemia by PIO was obtained without affecting levels of plasmatic insulin. A possible explanation for this effect is that PIO may have acted, as previously reported, on modifying insulin sensitivity in the liver. ${ }^{40}$ This mechanism can possibly explain the lower increase in body weight in PIO-treated PS1-KI females when compared with treated males of the same genotype.

Deregulation of glycemia is a key contributor to the development of microvascular DM complications. ${ }^{41}$ Altered glycemia is also strongly linked to the production of $\mathrm{mROS}^{42}$ and affects cognition. ${ }^{43}$ 

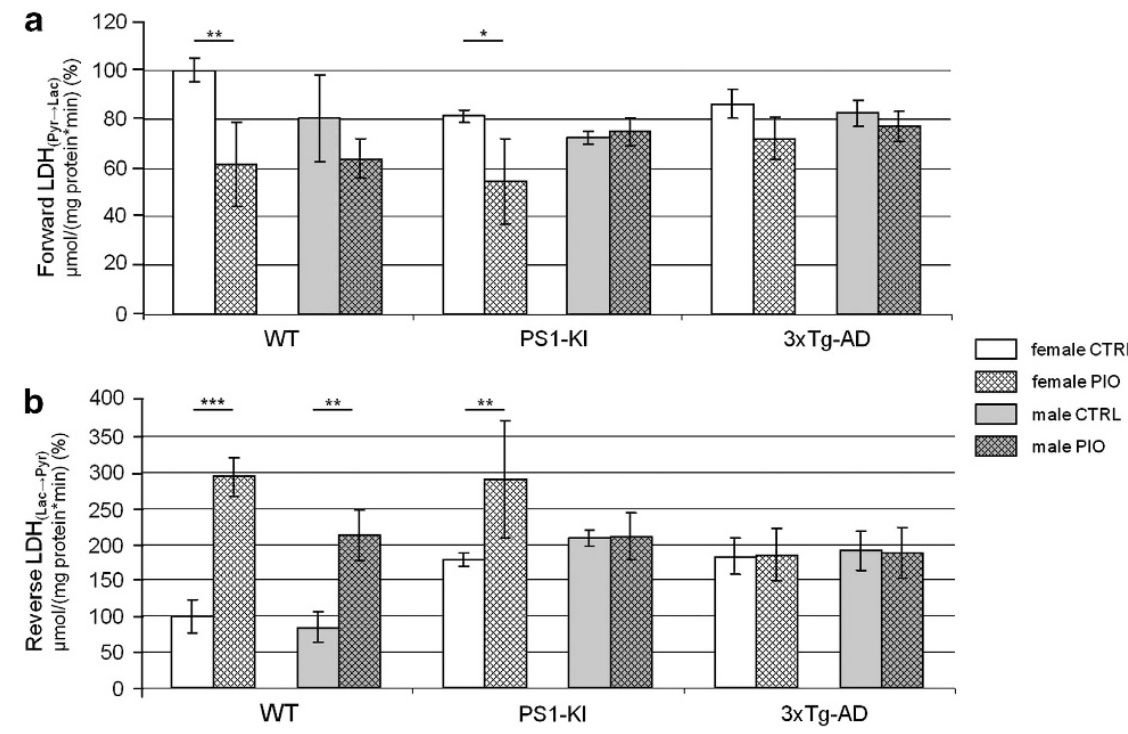

Figure 7 PIO effect on LDH activities. Cytosolic samples from whole hemispheres of treated and untreated animals of the three genotypes were evaluated for (a) forward and (b) reverse LDH activities. (a) Pyruvate reductase ( $\mathrm{LDH}_{\text {Pyr } \rightarrow \text { Lac }}$ ) activity. PIO treatment induced significant reduction of forward LDH activity in WT female mice; a trend toward decreased LDH activity was found in PIO-diet PS1-KI females. (b) Lactate oxidase ( $\mathrm{LDH}_{\mathrm{Lac} \rightarrow \mathrm{Pyr}}$ ) activity. In both male and female WT mice, PIO treatment increased reverse LDH activity. Significant increase in reverse LDH activity is present in PIO-diet PS1-KI females. Data are shown as percentage of LDH activities found in CTRL-diet female WT mice, a group used as control. Bars represent means \pm S.E.M.; $n=4$. Statistical analysis was performed with analysis of variance for repeated measures. ${ }^{*} 0.05<P \leq 0.095 ;{ }^{* *} 0.05 \leq P<0.01 ;{ }^{* *} P \leq 0.01$

In our study, we investigated the role of mitochondria in the cognitive effects of PIO and studied complexes I and IV expression levels and activity as these complexes are known to be modulated by TZDs. ${ }^{44,45}$

$\mathrm{NADH}$ dehydrogenase at complex I and the interface between ubiquinone and complex III are principal electron transport chain sites where electrons can react with molecular oxygen and yield superoxide anions that can be converted to other neurotoxic ROS. ${ }^{46}$

Complex IV is a multimeric enzyme that not anly catalyzes the last step of respiration but also contributes to the storage of energy in the form of an electrochemical gradient used by the oxidative phosphorylation to synthesize ATP. Thus, changes in complex IV activity may affect ATP production from mitochondria, a process that in some circumstances can lead to compensatory increases in anaerobic glycolysis and ultimately produces high lactate levels.

This metabolic shift is potentially relevant for cognition as lactate is the primary energetic source for the brain. Lactate is provided by astrocytes, brain cells that store glucose in the form of glycogen and, on demand, supply this substrate to neurons, ${ }^{47}$ a process that favors hippocampal long-term potentiation. ${ }^{48}$ To clarify whether PIO treatment affected lactate levels, we evaluated forward $\left(\mathrm{LDH}_{\mathrm{Pyr} \rightarrow \mathrm{Lac}}\right)$ and reverse $\left(\mathrm{LDH}_{\mathrm{Lac} \rightarrow \text { Pyr }}\right) \mathrm{LDH}$ activities in brain samples from all the study groups.

We found increased reverse $\left(\mathrm{LDH}_{\mathrm{Lac} \rightarrow \text { Pyr }}\right)$ activity in PIOtreated PS1-KI females, a process likely leading to increased pyruvate levels. These results are not in line with previous finding in cultured astrocytes where PIO exposure was found to inhibit pyruvate-driven respiration and increase anaerobic glycolysis. $^{49}$

Combining LDH and mitochondrial effects of PIO on PS1-KI females (the only group showing cognitive impairment, a process indeed counteracted by treatment), a speculative protective pathway, centered on oxidative stress, can be hypothesized. The potential (as suggested by the trend we found) PIO-driven inhibition of complex I (leading to reduced generation of mtROS) along with increased reverse LDH activity may ultimately promote decreased oxidative stress if one considers that pyruvate and $\mathrm{NADH}$ have known antioxidant properties. ${ }^{50,51}$ Ultimately, this reduction of ROS levels can impinge on cognition as free radicals have been found to modulate excitability. ${ }^{52}$

In WT male mice, PIO treatment also enhanced short-term memory as assessed by MWM, an effect that goes along with modifications of complex I and LDH activity that are similar to the ones observed in PS1-KI females, thereby suggesting a possible common mechanism of action. However, ORT data showed a trend toward worsening of memory performance in the same group, making the overall net cognitive effect of PIO difficult to interpret.

In our study, PIO treatment did not show improvement of memory performances in pre-symptomatic 3xTg-AD mice. During the preparation of the manuscript, a study testing PIO treatment (4 month; $8 \mathrm{mg} / \mathrm{kg} /$ day) started on 10-month-old female 3xTg-AD mice indicated enhanced learning assessed by one-way active avoidance test. ${ }^{53}$ Discrepancies between the two studies can be ascribed to different timing and length of the treatments (10-month-old mice/4-month treatment for the Searcy et al.'s study versus 3-month-old mice/9-month treatment in our case), differences in levels of cognitive decline (fully developed in the Searcy et al.'s case, absent in our study) as well as differences in the tests used to evaluate cognitive performances (one-way active avoidance for Searcy et al., MWM in our case).

Our findings are partially in line with a study investigating $\mathrm{PIO}$ effects (administered for $6-8$ weeks at $20 \mathrm{mg} / \mathrm{kg} /$ day by 
food) in 14-month-old hAPP swe-ind mice. ${ }^{54}$ The study showed significant attenuation of astroglial activation as well as normalization of cerebral blood flow and cerebral glucose utilization in response to increased neuronal activity but found no effects on spatial memory performances.

Our results are not in line with a study showing that rosiglitazone (ROSI, another TZD) completely rescued cognitive impairment in old Tg AD mice (hAPP swe-ind $_{\text {) and }}$ prevented the development of early cognitive impairment in young animals of the same genotype. ${ }^{55}$ Differences between the two studies can be related to genotypes hAPP $_{\text {swe-ind }}$ are engineered to only develop amyloid-dependent pathology while our model shows also tau-dependent pathology), drug regimen (4-week treatment by gavage at $5 \mathrm{mg} / \mathrm{kg} /$ day instead of a 9-month treatment of $20 \mathrm{mg} / \mathrm{kg} /$ day administered by food), and used TZD (ROSI instead of PIO). ROSI and PIO have similar effects on glycemic control, ${ }^{56}$ but, compared with ROSI, PIO has a more pronounced beneficial effect on plasmatic lipidic profile. ${ }^{57}$ Differences in efficacy between the two TZDs are also likely related to the higher PPAR $\alpha$ agonist activity of $\mathrm{PIO},{ }^{58}$ a mechanism responsible for modulation of mitochondrial functioning. ${ }^{59}$

\section{Conclusions}

Overall, our study indicates that $\mathrm{PIO}$ is able to promote cognitive-enhancing effects in a female population of mutant PS1-KI-mice showing signs of cognitive deficits. These findings suggest that the drug can favor cognition in a disease-prone transgenic background. Cognitive-enhancing effects observed in PS1-KI animals but not in 3xTg-AD mice possibly indicate the presence of a cognitive reserve lost in the pre-symptomatic 3xTg-AD but still present in PS1-KI mice.

These results warrant further investigation to explore whether PIO beneficial activities can be extended and have translational value in FAD patients carrying PS1 mutations. A larger cohort study sample is also needed to clarify whether PIO can exert pro-cognitive actions in aging WT animals.

\footnotetext{
Materials and Methods

Animals and treatment paradigm. Procedures involving animals and their care were approved by the institutional Ethics Committee (CeSI protocol No.: AD-301) and conducted in conformity with the institutional guidelines that are in compliance with national (D.L. n. 116, G.U., suppl. 40, 18 February 1992) and international laws and policies. All efforts were made to minimize the number of animals used and their suffering. PS1-KI mice used in the study harbor the FADlinked mutation of presenilin $1, \mathrm{PS}_{\mathrm{M} 146 \mathrm{~V}}$, and were originally generated as a hybrid 129/C57BL6 background. ${ }^{60}$ Triple-transgenic mice, the 3xTg-AD, overexpressing mutant APP $\left(\mathrm{APP}_{\mathrm{Swe}}\right), \mathrm{PS} 1$ (PS1 $\left._{\mathrm{M} 146 \mathrm{~V}}\right)$, and hyper-phosphorylated tau $\left(\operatorname{tau}_{P 301 \mathrm{~L}}\right)$, were originally generated by coinjecting two independent transgene constructs encoding human APP $_{\text {Swe }}$ and tau $\mathrm{P}_{301 \mathrm{~L}}(4 \mathrm{R} / \mathrm{ON}$ ) (controlled by murine Thy1.2 regulatory elements) in single-cell embryos harvested from mutant homozygous PS1 ${ }_{\mathrm{M} 146 \mathrm{~V}}$ knock-in mice. These mice have been characterized and described by Oddo et al. ${ }^{26}$ and generously provided by Frank Laferla. WT mice of mixed genetic background 129/C57BL6 were used as controls. Twenty-four animals (24 females and 24 males) for each genotype group (3xTg-AD, PS1-KI, and WT) were enrolled at 3 months of age in the study and randomly assigned to either a control (CTRL; $n=12$ per group) or PIO-containing (PIO; $n=12$ per group) diet for 9 months. Pioglitazone Actos (Takeda Pharmaceuticals, Osaka, Japan) was added to standard mice chow at 40 p.p.m. yielding a final dose of $20 \mathrm{mg} / \mathrm{kg}$ body weight/day.
}

MWM. The MWM apparatus consists of a circular plastic tank (1.3 $\mathrm{m}$ diameter) filled with water. The maze was located in a room containing several intra and extra-maze visual cues. A visible platform was used at the beginning of the MWM test to make sure animals had no vision deficits. Mice were then trained to swim in the tank and climb on a $(12 \times 13 \mathrm{~cm})$ rectangular platform submerged $2 \mathrm{~cm}$ beneath the surface of the water. Mice were given four trials per day for 4 consecutive days. To reduce the stress related to the task, mice were placed on the platform $10 \mathrm{~s}$ before the beginning of the first training session. If a mouse failed to find the platform within $90 \mathrm{~s}$, it was manually guided to the platform and allowed to remain there for $10 \mathrm{~s}$. Between trials, mice were placed back into a holding cage under a warming fan for $20 \mathrm{~min}$. Retention of the spatial memory task was assessed at 1.5 and $24 \mathrm{~h}$ after the end of the last training trial. Both probe trials consisted of a 60-s free swim in the pool without the platform, that were videotaped for subsequent analysis manually performed by two operators who were in blind conditions with respect to treatments. Parameters measured during the probe trial included: (1) time spent to reach and cross the platform location (latency) and (2) the number of crosses over the platform location (crosses).

ORT. The one-trial object-place recognition test was administered to all mice in the study. Briefly, two plexiglass $(30 \times 15 \mathrm{~cm})$ cages were used for the whole experiment. In the habituation phase, mice, one per cage, were placed for $10 \mathrm{~min}$ per day for 2 consecutive days in empty cages. On day 3, the mouse was placed in a cage containing two identical objects spaced $\sim 15 \mathrm{~cm}$ apart and allowed to explore the objects for $8 \mathrm{~min}$ before returning to the home cage. To rule out innate preferences, all objects were separately tested on mice that were not part of the study. Objects were always thoroughly cleaned before each trial. On day 4, the mouse was returned to the experimental cage containing objects that have been previously presented, one of them was left in the previous location (familiar location) while the other one was moved in a new location (novel location). Mice were then allowed to explore the two objects during a 5-min-probe test that was videotaped for subsequent analysis manually performed by two operators in blind conditions. Scoring of the object-place recognition performance was based on time spent to explore both objects, presented in familiar location and in novel one. The behavior of the mouse was considered explorative (i.e. exploration) when the animal head was within $1 \mathrm{~cm}$ from the object with the neck extended and vibrissae moving. Simple proximity or chewing did not count as exploration. Thus, a DI was calculated, applying the following formula: $((A-B) /(A+B)) \times 100$, where $A$ is the time spent to explore the object in the novel location and $B$ is the time spent on the object in the familiar one. ${ }^{32}$

Metabolic measurements. Measurement of food intake, body weight, and blood glucose levels (fasting glycemia) were carried out every week during the 9month treatment period. Fasting glycemia was measured from a drop of blood obtained from tail tip using a commercial glucometer (Accu-Chek Compact Plus; Roche Applied Science, Roche Diagnostic SpA, Monza, Italy) following the manufacturer's instructions.

IPGTT. IPGTT was performed, once a month, after $16-17 \mathrm{~h}$ of fasting. Glucose $(1 \mathrm{~g} / \mathrm{kg}$ body weight; $20 \%$ glucose solution) was administered by intraperitoneal injection. Blood glucose levels were measured before $(0 \mathrm{~min})$ and $5,15,30,45$, 60,120 , and $180 \mathrm{~min}$ after injection. Blood samples were taken from the tail vein and blood glucose levels were measured using a glucometer. Glucose tolerance was calculated as area under the curve during the 3-h period.

Serum insulin levels. Fasting serum insulin levels were determined by collecting, once a month, blood samples from a tail vein. Serum samples were obtained by centrifuging blood samples at 5000 r.p.m. for $30 \mathrm{~min}$ at $+4{ }^{\circ} \mathrm{C}$ (Highperformance Microcentrifuge 5417 R, Eppendorf, Milan, Italy). Serum insulin was then determined by ELISA (Ultrasensitive Mouse Insulin ELISA kit, Mercodia, Test Medical, Zola Pedrosa, Italy) following the manufacturer's instructions.

Analysis of mitochondrial complexes I and IV activity and expression. At the end of treatment and after behavioral analysis, mice were killed by carbon dioxide inhalation and following cervical dislocation, and tissue samples were collected. Brains were rapidly taken after removal of cerebellum and sagittally halved in two hemispheres. Samples were immediately frozen with liquid nitrogen and kept at $-80^{\circ} \mathrm{C}$ for subsequent analysis. Hippocampi and cerebral cortices from four animals per group were quickly dissected on ice and separately frozen, first by liquid nitrogen, then kept at $-80^{\circ} \mathrm{C}$ for BN-PAGE analysis of mitochondrial enzymatic activity. Samples for BN-PAGE were prepared as 
previously described ${ }^{35}$ with minor modifications. Briefly, hippocampal and cortical tissues (10 mg; wet weight) were homogenized in BN-sample buffer $1(250 \mathrm{mM}$ Sucrose, $30 \mathrm{mM}$ morpholine-propane sulfonate buffer, $0.2 \mathrm{mM}$ phenylmethylsulfonyl fluoride, pH 7.2) using a homogenizer with a tight-fitting Teflon pestle ( $60 \mathrm{sec}$ at 500 r.p.m.) kept cold. Homogenates were centrifuged at $20000 \times \mathrm{g}$ for $20 \mathrm{~min}$ at $4{ }^{\circ} \mathrm{C}$ and supernatants discarded. To solubilize mitochondrial membranes, mitochondria-rich pellets were re-suspended in BN-sample buffer $2(1 \mathrm{M}$ aminocaproic acid, $50 \mathrm{mM}$ Bis-Tris- $\mathrm{HCl}$ buffer, $\mathrm{pH}$ 7), homogenized by twirling with a tiny spatula and then incubated for $5 \mathrm{~min}$ at $4{ }^{\circ} \mathrm{C}$ with freshly prepared $10 \%$ solution of dodecyl maltoside (to solubilize individual respiratory chain complexes). Homogenates were centrifuged at $20000 \times g$ for $1 \mathrm{~h}$ at $4^{\circ} \mathrm{C}$. Supernatants were collected and protein quantification was performed using the Bradford method. Next, Coomassie-G250 dye (5\% in $1 \mathrm{M}$ aminocaproic acid) was added to samples (at a ratio of $1: 6$, respectively) and they were loaded into three $6-13 \%$ gradient acrylamide gels for BN-PAGE. After electrophoresis, to evaluate the amount of respiratory chain complexes I and IV, one gel was fixed in $40 \%$ methanol and stained overnight with a Coomassie Blue Solution (Brillant Blue $\mathrm{G}$ and methanol used at a ratio of $1: 4$, respectively). Following the staining, gels were de-stained with 10\% acetic acid and $25 \%$ methanol for $1 \mathrm{~min}$ and washed with $25 \%$ methanol. Band areas were expressed as absolute values (arbitrary units). For evaluation of complex I (NADH-dehydrogenase) activity, the second gel was incubated with $0.1 \mathrm{M}$ Tris- $\mathrm{HCl}, 768 \mathrm{mM}$ glycine, $0.1 \mathrm{mM} \beta-\mathrm{NADH}$ and $0.04 \%$ Nitro Blue Tetrazolium pH 7.4 at room temperature; for complex IV (COX) activity, the third gel was incubated with $5 \mathrm{mg}$ of $3,3^{\prime}$-diaminobenzidine tetrahydrochloride dissolved in $9 \mathrm{ml}$ phosphate buffer $(0.05 \mathrm{M} \mathrm{pH} 7.4), 1 \mathrm{ml}$ catalase $(20 \mathrm{mg} / \mathrm{ml})$, $10 \mathrm{mg}$ cytocrome $\mathrm{C}$, and $750 \mathrm{mg}$ sucrose at room temperature. Violet-colored complex I bands and red-stained complex IV bands were measured using the BioRad Imaging Densitometer (Quantity One Analysis Software, Bio-Rad, Hercules, CA, USA) with a blue filter inserted to minimize interference from the residual Coomassie Blue. Band areas were expressed as absolute values (arbitrary units) and optical densities of bands from each loading amount were plotted against its respective value determined in Coomassie Blue gel.

Analysis of cytosolic LDH activities. LDH activities converting pyruvate (Pyr) to lactate (Lac) ( $\left.\mathrm{LDH}_{\text {Pyr } \rightarrow \mathrm{Lac}}\right)$ as well as $\mathrm{Lac}$ to Pyr $\left(\mathrm{LDH}_{\mathrm{Lac} \rightarrow \text { Pyr }}\right)$ were spectrophotometrically assayed as previously described ${ }^{38}$ using a tunable microplate reader (Spectramax 190; Molecular Devices, Sunnyvale, CA, USA). Briefly, four hemispheres per group were homogenized in phosphate buffer solution $(20 \mathrm{ml} / \mathrm{g}$ of tissue, $0.010 \mathrm{M}, \mathrm{pH}=7.4$ ). Homogenates were centrifuged at $2000 \times g$ for $3 \mathrm{~min}$ at $4^{\circ} \mathrm{C}$ to remove cell debris and nuclear pellets. Supernatants were further centrifuged at $8000 \times g$ for $10 \mathrm{~min}$ at $4{ }^{\circ} \mathrm{C}$ in order to obtain the cytosolic fraction. Protein concentration was determined according to Bradford assay. For $\mathrm{LDH}_{\mathrm{Pyr} \rightarrow \mathrm{Lac}}$ activity determination, pyruvate $(25 \mu \mathrm{l}, 2.50 \mathrm{~g} / \mathrm{l})$ and reduced nicotinamide adenine dinucleotide (NADH, $100 \mu \mathrm{l}, 0.3 \mathrm{~g} / \mathrm{l})$ were added to the samples $(50 \mu \mathrm{l})$. Decrease of absorbance at $340 \mathrm{~nm}$ was measured at $25^{\circ} \mathrm{C}$ in 1-min intervals for $5 \mathrm{~min}$. For $\mathrm{LDH}_{\mathrm{Lac} \rightarrow \text { Pyr }}$ activity determination, lactate $\left(25 \mu \mathrm{l}, 8.6 \mathrm{~g} \mathrm{~L}^{-1}\right)$ and oxidized nicotinamide adenine dinucleotide (NAD $\left.{ }^{+}, 100 \mu l, 3.5 \mathrm{~g} / \mathrm{l}\right)$ were added to the sample $(25 \mu \mathrm{l})$ following the adjustment of $\mathrm{pH}$ to 8.8 with $0.05 \mathrm{M}$ sodium pyrophospate. Increase of absorbance at $340 \mathrm{~nm}$ was measured as described above. $\mathrm{LDH}_{\text {Pyr } \rightarrow \text { Lac }}$ and $\mathrm{LDH}_{\mathrm{Lac} \rightarrow \text { Pyr }}$ activities were expressed as $\mu \mathrm{mol} /($ protein $\mathrm{mg} \times \mathrm{min}$ ).

Statistical analysis. Metabolic and behavioral data were analyzed with analysis of variance for repeated measures. Statistical analyses were performed using SAS (SAS, 9.1.3 for Windows, Cary, NC; SAS Institute 1989). Data from brain mitochondrial complexes I and IV analysis were evaluated for statistical significance using the two-tailed Student's $t$-test for unpaired comparisons between the treated and the untreated groups (95\% confidence level). Significance of the differences for $\mathrm{LDH}_{\text {Pyr } \rightarrow \mathrm{Lac}}$ and $\mathrm{LDH}_{\mathrm{Lac} \rightarrow \text { Pyr }}$ activities between female and male WT, PS1-KI, and 3xTg-AD mice were assessed by three-way ANOVA followed by Fisher's least significant difference post-hoc test considering the following factors: genotype, gender, and treatment. Homogeneity of variance was previously assessed by Levene test. When data failed homogeneity of variance test, aligned rank transformation of data was performed using the ARTweb software available at http:/ffaculty.washington.edu/ aimgroup/proj/art/artweb/.

All data are expressed as mean \pm S.E.M.

\section{Conflict of Interest}

The authors declare no conflict of interest.
Acknowledgements. SLS is supported by funds from the Italian Department of Education (FIRB 2003; PRIN 2006; PRIN 2008).

1. Hoyer S. Senile dementia and Alzheimer's disease. Brain blood flow and metabolism. Prog Neuropsychopharmacol Biol Psychiatry 1986; 10: 447-478.

2. Ott A, Stolk RP, van Harskamp F, Pols HA, Hofman A, Breteler MM. Diabetes mellitus and the risk of dementia: The Rotterdam Study. Neurology 1999; 53: 1937-1942.

3. Schnaider Beeri M, Goldbourt U, Silverman JM, Noy S, Schmeidler J, Ravona-Springer R et al. Diabetes mellitus in midlife and the risk of dementia three decades later. Neurology 2004; 63: 1902-1907.

4. Craft S, Watson GS. Insulin and neurodegenerative disease: shared and specific mechanisms. Lancet Neurol 2004; 3: 169-178.

5. Frolich L, Blum-Degen D, Riederer P, Hoyer S. A disturbance in the neuronal insulin receptor signal transduction in sporadic Alzheimer's disease. Ann N Y Acad Sci 1999; 893: 290-293

6. Recio-Pinto E, Lang FF, Ishii DN. Insulin and insulin-like growth factor II permit nerve growth factor binding and the neurite formation response in cultured human neuroblastoma cells. Proc Natl Acad Sci USA 1984; 81: 2562-2566.

7. Baskin DG, Porte D Jr., Guest K, Dorsa DM. Regional concentrations of insulin in the rat brain. Endocrinology 1983; 112: 898-903.

8. Zhao W, Chen H, Xu H, Moore E, Meiri N, Quon MJ et al. Brain insulin receptors and spatial memory. Correlated changes in gene expression, tyrosine phosphorylation, and signaling molecules in the hippocampus of water maze trained rats. J Biol Chem 1999; 274: 34893-34902.

9. Das P, Parsons AD, Scarborough J, Hoffman J, Wilson J, Thompson RN et al. Electrophysiological and behavioral phenotype of insulin receptor defective mice. Physiol Behav 2005; 86: 287-296.

10. Francis GJ, Martinez JA, Liu WQ, Xu K, Ayer A, Fine J et al. Intranasal insulin prevents cognitive decline, cerebral atrophy and white matter changes in murine type I diabetic encephalopathy. Brain 2008; 131(Pt 12): 3311-3334.

11. Marks DR, Tucker K, Cavallin MA, Mast TG, Fadool DA. Awake intranasal insulin delivery modifies protein complexes and alters memory, anxiety, and olfactory behaviors. $J$ Neurosci 2009; 29: 6734-6751.

12. Craft S, Baker LD, Montine TJ, Minoshima S, Watson GS, Claxton A et al. Intranasal insulin therapy for Alzheimer disease and amnestic mild cognitive impairment: a pilot clinical trial. Arch Neurol 2012; 69: 29-38

13. Ott V, Benedict C, Schultes B, Born J, Hallschmid M. Intranasal administration of insulin to the brain impacts cognitive function and peripheral metabolism. Diabetes Obes Metab 2012; 14: 214-221.

14. de la Monte SM. Brain insulin resistance and deficiency as therapeutic targets in Alzheimer's disease. Curr Alzheimer Res 2012; 9: 35-66.

15. Sarruf DA, Yu F, Nguyen HT, Williams DL, Printz RL, Niswender KD et al. Expression of peroxisome proliferator-activated receptor-gamma in key neuronal subsets regulating glucose metabolism and energy homeostasis. Endocrinology 2009; 150: 707-712.

16. Nicolakakis N, Hamel E. The nuclear receptor PPARgamma as a therapeutic target for cerebrovascular and brain dysfunction in Alzheimer's disease. Front Aging Neurosci 2010; 2.

17. Zhao WQ, De Felice FG, Fernandez S, Chen H, Lambert MP, Quon MJ et al. Amyloid beta oligomers induce impairment of neuronal insulin receptors. FASEB J 2008; 22: 246-260.

18. De Felice FG, Vieira MN, Bomfim TR, Decker H, Velasco PT, Lambert MP et al. Protection of synapses against Alzheimer's-linked toxins: insulin signaling prevents the pathogenic binding of Abeta oligomers. Proc Natl Acad Sci USA 2009; 106: 1971-1976.

19. Sastre M, Dewachter I, Rossner S, Bogdanovic N, Rosen E, Borghgraef $P$ et al. Nonsteroidal anti-inflammatory drugs repress beta-secretase gene promoter activity by the activation of PPARgamma. Proc Natl Acad Sci USA 2006; 103: 443-448.

20. Sastre M, Dewachter I, Landreth GE, Willson TM, Klockgether T, van Leuven F et al. Nonsteroidal anti-inflammatory drugs and peroxisome proliferator-activated receptorgamma agonists modulate immunostimulated processing of amyloid precursor protein through regulation of beta-secretase. J Neurosci 2003; 23: 9796-9804.

21. Swerdlow RH, Khan SM. A "mitochondrial cascade hypothesis" for sporadic Alzheimer's disease. Med Hypotheses 2004; 63: 8-20.

22. Colca JR, McDonald WG, Waldon DJ, Leone JW, Lull JM, Bannow CA et al. Identification of a novel mitochondrial protein ("mitoNEET") cross-linked specifically by a thiazolidinedione photoprobe. Am J Physiol Endocrinol Metab 2004; 286: E252-E260.

23. Kukidome D, Nishikawa T, Sonoda K, Imoto K, Fujisawa K, Yano M et al. Activation of AMP-activated protein kinase reduces hyperglycemia-induced mitochondrial reactive oxygen species production and promotes mitochondrial biogenesis in human umbilical vein endothelial cells. Diabetes 2006; 55: 120-127.

24. Elder GA, Gama Sosa MA, De Gasperi R, Dickstein DL, Hof PR. Presenilin transgenic mice as models of Alzheimer's disease. Brain Struct Funct 2010; 214: 127-143.

25. Auffret A, Gautheron V, Repici M, Kraftsik R, Mount HT, Mariani J et al. Age-dependent impairment of spine morphology and synaptic plasticity in hippocampal CA1 neurons of a presenilin 1 transgenic mouse model of Alzheimer's disease. J Neurosci 2009; 29: 10144-10152.

26. Oddo S, Caccamo A, Shepherd JD, Murphy MP, Golde TE, Kayed R et al. Tripletransgenic model of Alzheimer's disease with plaques and tangles: intracellular Abeta and synaptic dysfunction. Neuron 2003; 39: 409-421. 
27. Heneka MT, Sastre M, Dumitrescu-Ozimek L, Hanke A, Dewachter I, Kuiperi C et al. Acute treatment with the PPARgamma agonist pioglitazone and ibuprofen reduces glial inflammation and Abeta1-42 levels in APPV717I transgenic mice. Brain 2005; 128(Pt 6): 1442-1453.

28. Morris R. Developments of a water-maze procedure for studying spatial learning in the rat. J Neurosci Methods 1984; 11: 47-60.

29. Mumby DG, Gaskin S, Glenn MJ, Schramek TE, Lehmann H. Hippocampal damage and exploratory preferences in rats: memory for objects, places, and contexts. Learn Mem 2002; 9: 49-57.

30. Dere E, Huston JP, De Souza Silva MA. The pharmacology, neuroanatomy and neurogenetics of one-trial object recognition in rodents. Neurosci Biobehav Rev 2007; 31: 673-704.

31. Akkerman S, Blokland A, Reneerkens O, van Goethem NP, Bollen E, Gijselaers HJ et al. Object recognition testing: methodological considerations on exploration and discrimination measures. Behav Brain Res 2012; 232: 335-347.

32. Stefanko DP, Barrett RM, Ly AR, Reolon GK, Wood MA. Modulation of long-term memory for object recognition via HDAC inhibition. Proc Natl Acad Sci USA 2009; 106 9447-9452.

33. Kliewer SA, Forman BM, Blumberg B, Ong ES, Borgmeyer U, Mangelsdorf DJ et al. Differential expression and activation of a family of murine peroxisome proliferatoractivated receptors. Proc Natl Acad Sci USA 1994; 91: 7355-7359.

34. Mammucari $C$, Rizzuto R. Signaling pathways in mitochondrial dysfunction and aging. Mech Ageing Dev 2010; 131: 536-543.

35. Schagger $\mathrm{H}$. Electrophoretic techniques for isolation and quantification of oxidative phosphorylation complexes from human tissues. Methods Enzymol 1996; 264: 555-566.

36. Wittig I, Schagger H. Features and applications of blue-native and clear-native electrophoresis. Proteomics 2008; 8: 3974-3990.

37. Schagger $\mathrm{H}$, von Jagow $\mathrm{G}$. Blue native electrophoresis for isolation of membrane protein complexes in enzymatically active form. Anal Biochem 1991; 199: 223-231.

38. Krieg AF, Rosenblum LJ, Henry JB. Lactate dehydrogenase isoenzymes a comparison of pyruvate-to-lactate and lactate-to-pyruvate assays. Clin Chem 1967; 13: 196-203.

39. Ayala JE, Samuel VT, Morton GJ, Obici S, Croniger CM, Shulman Gl et al. Standard operating procedures for describing and performing metabolic tests of glucose homeostasis in mice. Dis Model Mech 2010; 3: 525-534.

40. Hsiao G, Chapman J, Ofrecio JM, Wilkes J, Resnik JL, Thapar D et al. Multi-tissue, selective PPARgamma modulation of insulin sensitivity and metabolic pathways in obese rats. Am J Physiol Endocrinol Metab 2011; 300: E164-E174.

41. The Diabetes Control and Complications Trial Research Group. The effect of intensive treatment of diabetes on the development and progression of long-term complications in insulin-dependent diabetes mellitus. N Engl J Med 1993; 329: 977-986.

42. Nishikawa T, Edelstein D, Brownlee M. The missing link: a single unifying mechanism for diabetic complications. Kidney Int Supp/ 2000; 77: S26-S30

43. Aviles-Olmos I, Limousin P, Lees A, Foltynie T. Parkinson's disease, insulin resistance and novel agents of neuroprotection. Brain; e-pub ahead of print 17 February 2012.

44. Brunmair B, Staniek K, Gras F, Scharf N, Althaym A, Clara R et al. Thiazolidinediones, like metformin, inhibit respiratory complex I: a common mechanism contributing to their antidiabetic actions? Diabetes 2004; 53: 1052-1059.

45. Ghosh S, Patel N, Rahn D, McAllister J, Sadeghi S, Horwitz G et al. The thiazolidinedione pioglitazone alters mitochondrial function in human neuron-like cells. Mol Pharmacol 2007; 71: $1695-1702$.

46. Balaban RS, Nemoto S, Finkel T. Mitochondria, oxidants, and aging. Cell 2005; 120 : 483-495.

47. Pellerin L, Magistretti PJ. Glutamate uptake into astrocytes stimulates aerobic glycolysis: a mechanism coupling neuronal activity to glucose utilization. Proc Natl Acad Sci USA 1994; 91: $10625-10629$.

48. Suzuki A, Stern SA, Bozdagi O, Huntley GW, Walker RH, Magistretti PJ et al. Astrocyte-neuron lactate transport is required for long-term memory formation. Cell 2011; 144: 810-823.

49. Dello Russo C, Gavrilyuk V, Weinberg G, Almeida A, Bolanos JP, Palmer J et al. Peroxisome proliferator-activated receptor gamma thiazolidinedione agonists increase glucose metabolism in astrocytes. J Biol Chem 2003; 278: 5828-5836.

50. Nakamichi N, Kambe Y, Oikawa H, Ogura M, Takano K, Tamaki K et al. Protection by exogenous pyruvate through a mechanism related to monocarboxylate transporters against cell death induced by hydrogen peroxide in cultured rat cortical neurons. J Neurochem 2005; 93: 84-93.

51. Zeng J, Yang GY, Ying W, Kelly M, Hirai K, James TL et al. Pyruvate improves recovery after PARP-1-associated energy failure induced by oxidative stress in neonatal rat cerebrocortical slices. J Cereb Blood Flow Metab 2007; 27: 304-315.

52. Wang TA, Yu YV, Govindaiah G, Ye X, Artinian L, Coleman TP et al. Circadian rhythm of redox state regulates excitability in suprachiasmatic nucleus neurons. Science 2012; 337: 839-842.

53. Searcy JL, Phelps JT, Pancani T, Kadish I, Popovic J, Anderson KL et al. Long-term pioglitazone treatment improves learning and attenuates pathological markers in a mouse model of Alzheimer's disease. J Alzheimers Dis 2012; 30: 943-961.

54. Nicolakakis N, Aboulkassim T, Ongali B, Lecrux C, Fernandes P, Rosa-Neto P et al. Complete rescue of cerebrovascular function in aged Alzheimer's disease transgenic mice by antioxidants and pioglitazone, a peroxisome proliferator-activated receptor gamma agonist. J Neurosci 2008; 28: 9287-9296.

55. Escribano L, Simon AM, Perez-Mediavilla A, Salazar-Colocho P, Del Rio J, Frechilla D. Rosiglitazone reverses memory decline and hippocampal glucocorticoid receptor downregulation in an Alzheimer's disease mouse model. Biochem Biophys Res Commun 2009; 379: 406-410.

56. Norris SL, Carson S, Roberts C. Comparative effectiveness of pioglitazone and rosiglitazone in type 2 diabetes, prediabetes, and the metabolic syndrome: a metaanalysis. Curr Diabetes Rev 2007; 3: 127-140.

57. Goldberg RB, Kendall DM, Deeg MA, Buse JB, Zagar AJ, Pinaire JA et al. A comparison of lipid and glycemic effects of pioglitazone and rosiglitazone in patients with type 2 diabetes and dyslipidemia. Diabetes Care 2005; 28: 1547-1554.

58. Duran-Sandoval D, Thomas AC, Bailleul B, Fruchart JC, Staels B. [Pharmacology of PPARalpha, PPARgamma and dual PPARalpha/gamma agonists in clinical development]. Med Sci (Paris) 2003; 19: 819-825.

59. Rabol R, Boushel R, Almdal T, Hansen CN, Ploug T, Haugaard SB et al. Opposite effects of pioglitazone and rosiglitazone on mitochondrial respiration in skeletal muscle of patients with type 2 diabetes. Diabetes Obes Metab 2010; 12: 806-814.

60. Guo Q, Fu W, Sopher BL, Miller MW, Ware CB, Martin GM et al. Increased vulnerability of hippocampal neurons to excitotoxic necrosis in presenilin-1 mutant knock-in mice. Nat Med 1999; 5: 101-106.

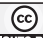

OMERTHHIS

published by Nature Publishing Group. This work is licensed under the Creative Commons Attribution-NonCommercial-No Derivative Works 3.0 Unported License. To view a copy of this license, visit http://creativecommons.org/licenses/by-nc-nd/3.0/

Supplementary Information accompanies the paper on Cell Death and Disease website (http://www.nature.com/cddis) 